\title{
Erros cometidos por estudantes de Odontologia de uma universidade pública brasileira na realização de radiografias periapicais
}

João Marcos Freitas Silva*, Lucas Caetano de Oliveira*, Breno Gonçalves Daroz*, Priscila Dias Peyneau**, Teresa Cristina Rangel Pereira**, Sergio Lins de-Azevedo-Vaz***

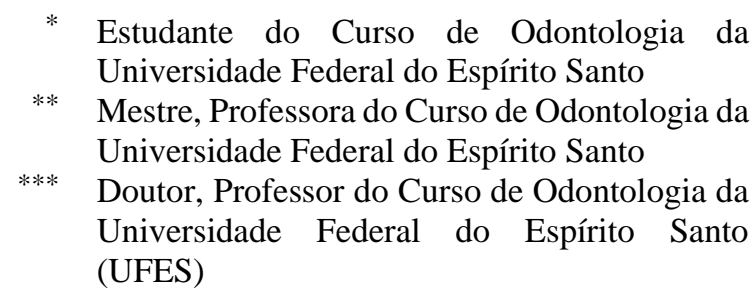

\section{RESUMO}

A proposta do estudo foi identificar e quantificar os erros mais cometidos por estudantes de Odontologia da UFES na execução de radiografias periapicais. Foi um estudo observacional com delineamento transversal cuja amostra constou de 352 radiografias contidas em prontuários de pacientes atendidos no curso nos últimos 5 anos. Os dados foram quantificados de acordo com tipo de erro e período do curso e submetidos ao teste de Qui-quadrado com nível de significância adotado em $5 \%$. Constatou-se grande frequência de erros $(84,37 \%)$, sendo o $9^{\circ}$ período o que apresentou a maior frequência $(92,30 \%)$ e o $8^{\circ}$ período, a menor $(70,00 \%)(\mathrm{p}<0,05)$. O erro mais observado foi o armazenamento incorreto $(15,60 \%)$, até então não quantificado em ambiente acadêmico. Os outros dois erros mais frequentes foram a falta de enquadramento $(14,60 \%)$ e imagens com baixa densidade $(10,80 \%)$. Concluiu-se que o índice de erros é alto na amostra estudada e que se deve atentar para o armazenamento de radiografias periapicais no âmbito universitário.

Descritores: Estudantes de Odontologia. Radiografia Dentária. Controle de Qualidade.

\section{INTRODUÇÃO}

A radiografia é um dos exames complementares disponíveis para o processo de diagnóstico em Odontologia, somando informações aos dados clínicos e permitindo o reconhecimento de eventuais processos patológicos. Para que as informações obtidas nas radiografias sejam relevantes ao diagnóstico, é necessário que a técnica utilizada, seu processamento e armazenamento tenham sido feitos da maneira correta.

$\mathrm{Na}$ técnica radiográfica periapical o receptor de imagem é inserido dentro da cavidade bucal do paciente. Ela é utilizada quando se deseja obter o máximo de detalhes possíveis das regiões dentárias, especialmente na região de periápice e periodonto. Assim, a radiografia periapical é indicada para o conhecimento da forma anatômica dos condutos radiculares e número de raízes, avaliação de lesões periapicais, avaliação da crista óssea alveolar e região de furca radicular $^{1}$.

Erros durante a execução da técnica radiográfica ou durante o processamento da 
imagem resultam em imagens insatisfatórias, as quais podem gerar interpretações erradas quanto à tarefa de diagnóstico em questão ${ }^{2}$. A Organização Mundial de Saúde apontou que a baixa qualidade nas imagens radiográficas é responsável pela redução da certeza no diagnóstico $^{3}$. Da mesma maneira, o armazenamento incorreto das radiografias periapicais - isto é, identificação do paciente e data do exame, montagem em cartelas segundo a posição anatômica - também pode prejudicar sua adequada interpretação. Assim, radiografias com erros técnicos poderão causar uma série de problemas para o cirurgião-dentista e para o paciente, tais como exposição excessiva e desnecessária do paciente aos raios $\mathrm{X}$, aumento do tempo clínico e gastos prescindíveis ${ }^{2}$.

Matheus et al. $(2000)^{4}$, Kreich et al. $(2002)^{2}$ e Queiroga et al. $(2010)^{5}$ demonstraram que a frequência de erros radiográficos cometidos por estudantes de Odontologia é superior a $80 \%$. Comparativamente, Carvalho et al. (2009) ${ }^{6}$ apontaram um índice de radiografias com erros próximo a $50 \%$. Dentre os erros mais frequentes nesses estudos, destacam-se o posicionamento incorreto do filme, ranhuras, erros de processamento e angulação horizontal ou vertical incorreta. Não há um consenso na literatura a respeito de qual seja o tipo de erro mais frequente, embora os erros de técnica/execução preponderem aos erros de processamento na maioria dos estudos 5 .

Com o passar dos anos na graduação, a experiência tida pelo estudante até então pode acabar interferindo na maneira de aplicar e executar a técnica corretamente. Esse fato é preocupante, uma vez que os estudantes que cursam as disciplinas clínicas do curso estão mais próximos de concluir a graduação, podendo carregar esses vícios para a vida profissional. Essa realidade mostra-se, então, indesejável frente às atuais Diretrizes Curriculares Nacionais (Resolução CNE/CES $3 / 2002^{7}$ ) que visam "um perfil do egresso com formação generalista, humanista, crítica e reflexiva, para atuar em todos os níveis de atenção à saúde, com base no rigor técnico e científico". Sendo assim, o objetivo do presente estudo foi identificar e quantificar os erros mais frequentes nas radiografias periapicais realizadas por estudantes do curso de Odontologia da Universidade Federal do Espírito Santo (UFES).

\section{MATERIAL E MÉTODOS}

Esta pesquisa foi iniciada após a aprovação do protocolo de pesquisa pelo Comitê de Ética em Pesquisa do Centro de Ciências da Saúde da UFES (Parecer CEP/CCS/UFES no 733.570 de 30/07/2014).

Tratou-se de um estudo observa-cional com delineamento transversal, cuja amostra constou de radiografias contidas nos prontuários armazenados na Central de Prontuários do curso (dados secundários). Foram critérios de inclusão: radiografias de pacientes sem distinção de gênero e faixa etária, realizadas por estudantes nas clínicas do $4^{\circ}$ ao $10^{\circ}$ período do curso de Odontologia da UFES nos últimos 5 anos. Radiografias realizadas por professores ou outros profissionais, ou ainda que não tivessem sido realizadas na UFES foram excluídas da amostra.

Todas as radiografias incluídas na amostra foram produzidas com filmes radiográficos e processamento químico manual. Não foi possível determinar o método de processamento manual utilizado, isto é, se tempo-temperatura ou visual, embora o primeiro seja o recomendado pela Portaria $453 / 98^{8}$. 
O número amostral foi calculado utilizando a fórmula para populações infinitas e adotando frequência esperada de erros de $80 \%$, intervalo de confiança de $95 \%$, nível de significância de 5\% e amplitude de 5\%. Dentro desses parâmetros, um total de 352 radiografias periapicais homogeneamente distribuídas entre os sete períodos em estudo foram analisadas, resultando em cerca de 50 radiografias por período. As disciplinas clínicas cujas radiografias integraram a amostra do estudo foram selecionadas por conveniência, buscando-se diversificar as áreas de atuação de forma a obter uma amostra mais representativa (quadro 1). Um número máximo de três radiografias por prontuário foi adotado também como forma de diversificar a amostra.

Quadro 1. Disciplinas clínicas cujas radiografias integraram a amostra do estudo

\begin{tabular}{|c|c|c|}
\hline Período & Disciplina & Atividade principal \\
\hline $4^{\circ}$ & Estágio Clínico Interdisciplinar I & Semiologia \\
\hline $5^{\circ}$ & Cirurgia Buco-maxilo-facial I & Exodontia de dentes irrompidos \\
\hline $6^{\circ}$ & Odontologia Restauradora Clínica & Restaurações diretas \\
\hline $7^{\circ}$ & Clínica Integrada I & Clínica Odontológica Integrada \\
\hline $8^{\circ}$ & Interdisciplinaridade em Paciente Infantil & Odontopediatria \\
\hline $9^{\circ}$ & Clínica Integrada II & Clínica Odontológica Integrada \\
\hline $10^{\circ}$ & Clínica Integrada III & Clínica Odontológica Integrada \\
\hline
\end{tabular}

Três examinadores, estudantes do $6^{\circ}$ período, foram treinados pelos docentes responsáveis pelo conteúdo de Radiologia Odontológica da UFES para garantir a confiabilidade na coleta de dados e resultados finais. Ao início do estudo, todos os examinadores já haviam cumprido a disciplina de Radiologia Odontológica há cerca de um ano. O treinamento compreendeu quatro sessões utilizando 70 radiografias periapicais não integrantes da amostra do estudo (equivalente a cerca de $20 \%$ da amostra), nas quais os erros considerados no estudo (quadro 2) foram demonstrados e discutidos com os três examinadores simultaneamente. As sessões de treinamento aconteceram num mesmo ambiente com iluminação reduzida (inferior a 50 lux), utilizando negatoscópio, máscara negra e lupa. Dúvidas apresentadas pelos examinadores foram resolvidas para que a coleta de dados pudesse ser iniciada.
Após o treinamento, os examinadores analisaram outras 70 radiografias integrantes da amostra, de forma independente, sob as mesmas condições. A seguir, houve uma análise intraexaminadores e interexaminadores, bem como uma comparação entre os examinadores do estudo e os examinadores de referência (docentes de Radiologia Odontológica), utilizando o índice Kappa. A coleta de dados somente foi iniciada quando a reprodutibilidade intra e interexaminadores, bem como a comparação aos examinadores de referência atingiram excelente concordância $(\mathrm{k}>0,75)$.

As 352 radiografias selecionadas para o estudo primeiramente foram codificadas para permitir o sigilo quanto à identidade dos pacientes e estudantes que as executaram. Os erros identificados foram anotados em ficha de coleta de dados própria, bem como o período e data na qual a radiografia foi realizada. 
Erros cometidos por estudantes de Odontologia na realização de radiografias periapicais

Quadro 2. Erros considerados no estudo e prováveis causas

\begin{tabular}{|c|c|}
\hline Erro identificado & Provável causa \\
\hline Imagem alongada & Ângulo vertical do feixe de raios X menor do que o ideal \\
\hline Imagem encurtada & Ângulo vertical do feixe de raios X maior do que o ideal \\
\hline Sobreposição das faces proximais & Angulação horizontal do feixe de raios X incorreta \\
\hline Meia-lua ou halo & Erro de ponto de incidência \\
\hline Imagem da prótese & Não-remoção prévia de próteses removíveis \\
\hline Posicionamento incorreto do picote & Posicionamento do picote na região apical dos dentes \\
\hline Filme invertido (padrão de escamas) & Utilização da face não-ativa do filme \\
\hline Falta de nitidez & Movimentação durante a exposição ou filme dobrado \\
\hline Dupla exposição & Filme exposto aos raios X duas vezes \\
\hline Falta de enquadramento & Centralização incorreta dos dentes no filme \\
\hline Alta densidade & Tempo de exposição ou de revelação* acima do ideal ou \\
& distância foco-filme menor que o ideal \\
\hline Baixa densidade & Tempo de exposição ou de revelação* abaixo do ideal ou \\
& distância foco-filme maior que o ideal \\
\hline Presença de ranhuras & Injúrias à emulsão durante o processamento \\
\hline Impressão digital & Toque digital na emulsão durante o processamento \\
\hline Radiografia amarelada & Lavagem final insuficiente \\
\hline Outros erros de processamento & Manchas por contaminação dos químicos \\
\hline Velamento radiográfico & Densidade extra decorrente de exposição à luz \\
\hline Armazenamento incorreto & Falta de identificação ou montagem incorreta \\
\hline
\end{tabular}

*Etapa de revelação do processamento radiográfico

Duas semanas após a coleta dos dados do estudo ter sido finalizada, $20 \%$ da amostra foi reavaliada para nova mensuração da reprodutibilidade interexaminadores pelo índice Kappa, bem como nova comparação aos examinadores de referência.

Os dados foram tratados por estatística descritiva (número absoluto e percentual) e inferencial (teste de Qui-quadrado com nível de significância em 5\%), de acordo com os fatores "tipo de erro" e "período do curso". As análises estatísticas foram feitas no pacote estatístico SPSS versão $17^{\circledR}$ para Windows (SPSS, Chicago, IL).

\section{RESULTADOS}

Ao final do estudo, os valores de Kappa para reprodutibilidade interexaminadores foram todos superiores a 0,75 (concordância excelente), assim como para comparação aos examinadores de referência, validan-

do novamente os examinadores (tabela 1).

$\mathrm{Na}$ tabela 2 observa-se que a quantidade de radiografias com erros foi maior do que as sem erros em todos os períodos analisados, o que resultou em um total de 297 radiografias $(84,37 \%)$ com erros e apenas $55(15,63 \%)$ sem erros. Assim, no total da amostra, a frequência de radiografias com erros foi estatisticamente maior que a frequência de radiografias sem erros $(\mathrm{p}<$ $0,05)$.

Das radiografias que apresentaram erros, a maioria (63\%) possuía mais de um erro (tabela 3). O $5^{\circ}$ período apresentou a maior frequência de erros associados $(74,42 \%)$.

A tabela 4 demonstra a quantidade de erros por tipo e período. Os tipos de erros estão apresentados em ordem decrescente segundo suas frequências, isto é, do mais frequente para o menos frequente.

Revista da ABENO • 16 (1):99-109, 2016. 
Erros cometidos por estudantes de Odontologia na realização de radiografias periapicais

Tabela 1. Valores de Kappa para reprodutibilidade interexaminadores

\begin{tabular}{lccc}
\hline & Examinador 1 & Examinador 2 & Examinador 3 \\
\hline Examinador 2 & 0,761 & - & - \\
Examinador 3 & 0,767 & 0,766 & - \\
Examinadores de referência & 0,763 & 0,758 & 0,771 \\
\hline
\end{tabular}

Tabela 2. Quantidade de radiografias com erros e sem erros por período

\begin{tabular}{|c|c|c|c|c|}
\hline Período & $\begin{array}{c}\text { Com erros } \\
\text { n }(\%)\end{array}$ & $\begin{array}{l}\text { Sem erros } \\
\text { n }(\%)\end{array}$ & $\begin{array}{c}\text { Total de } \\
\text { radiografias } \\
n(\%)\end{array}$ & Valores $\chi^{2}$ e p \\
\hline $4^{\circ}$ & $39(81,25)$ & $9(18,75)$ & $48(100,00)$ & \\
\hline $5^{\circ}$ & $43(86,00)$ & $7(14,00)$ & $50(100,00)$ & \\
\hline $6^{\circ}$ & $46(88,46)$ & $6(11,54)$ & $52(100,00)$ & \\
\hline $7^{\circ}$ & $45(91,83)$ & $4(8,17)$ & $49(100,00)$ & $\chi^{2}=14.117$ \\
\hline $8^{\circ}$ & $35(70,00)$ & $15(30,00)$ & $50(100,00)$ & \\
\hline $9^{\circ}$ & $48(92,30)$ & $4(7,70)$ & $52(100,00)$ & \\
\hline $10^{\circ}$ & $41(80,39)$ & $10(19,61)$ & $51(100,00)$ & $p=0,02$ \\
\hline Total & $297(84,37)$ & $55(15,63)$ & $352(100,00)$ & \\
\hline
\end{tabular}

Tabela 3. Quantidade de radiografias com um e mais de um erro

\begin{tabular}{cccc}
\hline Período & $\begin{array}{c}\text { Um erro } \\
\mathbf{n}(\boldsymbol{\%})\end{array}$ & $\begin{array}{c}\text { Mais de um erro } \\
\mathbf{n}(\boldsymbol{\%})\end{array}$ & $\begin{array}{c}\text { Total de radiografias com erros } \\
\mathbf{n}(\boldsymbol{\%})\end{array}$ \\
\hline $4^{\circ}$ & $19(48,71)$ & $20(51,29)$ & $39(100,00)$ \\
\hline $5^{\circ}$ & $11(25,58)$ & $32(74,42)$ & $43(100,00)$ \\
\hline $6^{\circ}$ & $17(36,95)$ & $29(63,05)$ & $46(100,00)$ \\
\hline $7^{\circ}$ & $17(37,77)$ & $28(62,23)$ & $45(100,00)$ \\
\hline $8^{\circ}$ & $13(37,14)$ & $22(62,86)$ & $35(100,00)$ \\
\hline $9^{\circ}$ & $15(31,25)$ & $33(68,75)$ & $48(100,00)$ \\
\hline $10^{\circ}$ & $19(46,34)$ & $22(53,66)$ & $41(100,00)$ \\
\hline Total & $111(37,00)$ & $186(63,00)$ & $297(100,00)$ \\
\hline
\end{tabular}


Tabela 4. Quantidade de erros por tipo e período

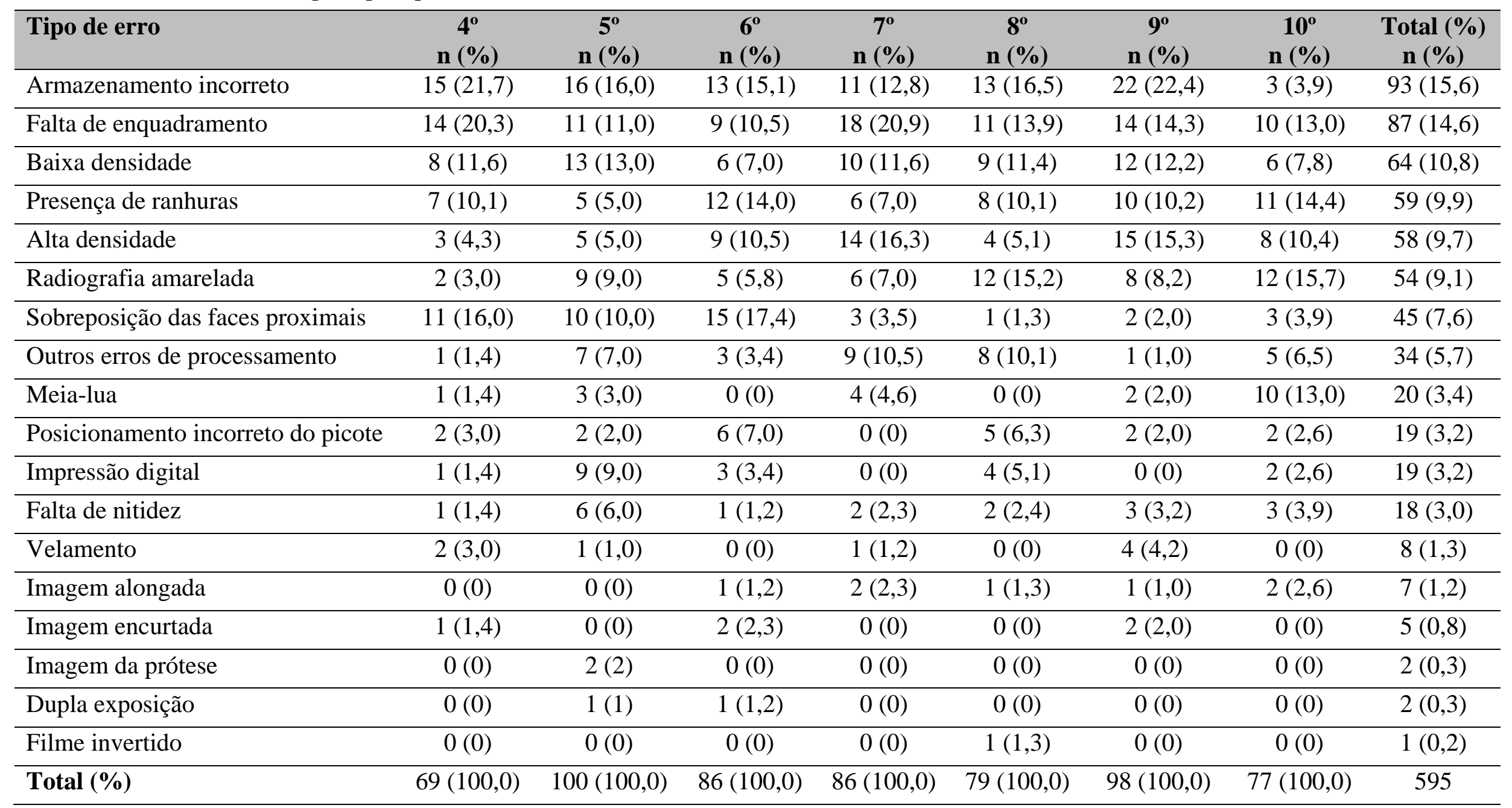




\section{DISCUSSÃO}

Erros de técnica e processamento radiográfico resultam em imagens inadequadas para o diagnóstico, podendo comprometer a interpretação radiográfica do cirurgião-dentista ${ }^{5}$. A interpretação radiográfica é um ato subjetivo e demanda a obtenção de imagens com qualidade aceitável, dentre seus pré-requisitos ${ }^{9}$. A radiografia ideal deveria possuir o máximo de detalhe, o mínimo de distorção e grau médio de densidade e contraste; demonstrando não somente os dentes da região, mas também estruturas vizinhas ${ }^{10}$. O armazenamento das radiografias identificadas e montadas em cartelas também deve ser realizado adequadamente, de forma que não haja prejuízo na sua visualização e interpretação.

Alguns fatores influenciam na produção das imagens radiográficas: fator energético (miliamperagem, tempo de exposição, quilovoltagem e distância focoreceptor), fator objeto (número atômico, densidade física e espessura do objeto), fator geométrico (tamanho da área focal, distâncias entre filme-objeto-fonte de raios $\mathrm{X}$, relações espaciais entre o objeto e filme), fator filme (tamanho da granulação, espessura da base e dupla emulsão) e fator processamento (condições da câmara escura e temperatura das soluções $)^{9}$.

Nenhuma imagem radiográfica digital foi incluída na amostra deste estudo tendo em vista que os sistemas digitais periapicais disponíveis na UFES ainda não são utilizados no atendimento clínico de pacientes.

Do total de 352 radiografias avaliadas no presente trabalho, 297 (84,37\%) apresentaram erros, resultado que se assemelha ao encontrado por Ribas et al. $(2008)^{11}(82 \%)$ e Queiroga et al. $(2010)^{5}$ $(82,51 \%)$. Por outro lado, Matheus et al. $(2000)^{4}(90.1 \%)$ e Kreich et al. $(2002)^{2}$
$(93,1 \%)$ observaram valores ainda maiores, enquanto Pontual et al. $(2005)^{12}(79,1 \%)$, Carvalho et al. $(2009)^{6}(49,49 \%)$ e Mendonça et al. $(2014)^{13} \quad(79,9 \%)$ encontraram percentuais menores de radiografias com erros. Embora existam tais variações, observa-se uma frequência majoritária de erros entre estudantes de graduação em Odontologia. Os tipos de erros avaliados nesses diferentes trabalhos são os mesmos de uma forma geral, muito embora suas frequências não sigam um mesmo padrão.

No presente estudo, 111 (37\%) das radiografias que apresentaram erros possuíam apenas um tipo de erro e 186 (63\%), mais de um. Gonçalves et al. (2003) ${ }^{14}$ e Fernandes et al. $(2010)^{3}$ também observaram que a maioria das radiografias analisadas em seus estudos apresentou mais de um tipo de erro. Contudo, radiografias produzidas durante o tratamento endodôntico constituíram as amostras desses estudos. Interessante mencionar, também, que Fernandes et al. $(2010)^{3}$ encontraram uma frequência de radiografias com erros menor que a do presente estudo $(57,7 \%)$.

Dados referentes a serviços de saúde e auditoria também estão disponíveis na literatura. Radiografias de um serviço de saúde finlandês foram avaliadas quanto a sua qualidade utilizando um escore de 0 a 9 , de acordo com a presença de erros. Apenas 13\% das radiografias periapicais analisadas apresentaram escore com valor máximo para qualidade $(9)^{15}$. Na auditoria de um plano de saúde odontológico brasileiro, os erros mais cometidos pelos conveniados foram alongamento, baixa densidade e halo/meia-lua ${ }^{16}$.

Ao se analisar a frequência percentual de erros entre os períodos, encontrou-se diferença estatisticamente significante. $\mathrm{O}$ desenho do estudo não permitiu comparações pareadas entre os períodos, sendo o teste de Qui-quadrado realizado de forma explo16 (1):99-109, 2016. 
ratória. Acredita-se que a diferença estatística se deva à menor taxa de erros observada no $8^{\circ}$ período em relação aos demais (70\%). Uma possível justificativa para esse resultado refere-se ao fato de a avaliação das radiografias do $8^{\circ}$ período ter sido feita na disciplina de Interdisciplinaridade em Paciente Infantil, na qual as técnicas empregadas diferem das técnicas-padrão. Pode-se inferir que os estudantes que se encontram nesse estágio do curso necessitaram revisitar conceitos anteriores das disciplinas de Radiologia e, como consequência desta atualização e revisão de conteúdos, melhorararam seu desempenho prático.

O $9^{\circ}$ período apresentou a maior taxa de erros do estudo $(92,30 \%)$. Acredita-se que a experiência dos estudantes durante a graduação possa ter interferido na maneira de aplicar e executar as técnicas de forma correta. Esse fato é preocupante uma vez que os estudantes que cursam as disciplinas clínicas do curso estão mais próximos de concluir a graduação e podem carregar esses vícios para a vida profissional. Desta forma, os profissionais egressos ficam mais sujeitos a falhas durante o tratamento odontológico.

De todos os erros encontrados na amostra, o mais frequente foi o armazenamento incorreto das radiografias $(15,6 \%)$, no qual considerou-se a falta de identificação do paciente, data de realização do exame ou montagem incorreta na cartela. $\mathrm{O} 9^{\circ}$ período foi o que mais apresentou esse tipo de erro $(22,4 \%)$. Na rotina clínica, radiografias sem identificação do paciente podem ser confundidas com exames de outro paciente. A ausência do registro da data de realização do exame dificulta a proservação e comparação com radiografias anteriores. Radiografias montadas incorretamente desorientam o profissional quanto à região radiografada, podendo resultar na realização inadvertida de procedimentos em dentes que não aqueles planejados.

Até o presente momento, não é de conhecimento dos autores que a frequência de erros de armazenamento de radiografias periapicais realizadas por estudantes de Odontologia tenha sido quantificada em algum outro estudo. Gonçalves et al. $(2003)^{14}$ encontraram uma grande ocorrência de erros de montagem em cartelas, especialmente na região de pré-molares inferiores; contudo os resultados não foram expressos sob frequência absoluta ou percentual. A amostra por eles avaliada também difere do presente estudo, uma vez que se referia a estudantes de graduação e pós-graduação na área de Endodontia. Helminem et al. $(2000)^{15}$ observaram baixa frequência de erros de armazenamento em radiografias produzidas num serviço de saúde finlandês, no qual falta de identificação da área radiografada foi o mais frequente $(54,0 \%)$. No presente estudo os erros de armazenamento não foram subdivididos.

Falta de enquadramento dos dentes no filme radiográfico foi o segundo erro mais frequente neste trabalho $(14,6 \%)$, o qual prevaleceu no $7^{\circ}$ período $(20,9 \%)$. Matheus et al. $(2000)^{4}$ e Mendonça et al. (2014) ${ }^{13}$ também apontaram esse erro como o segundo mais frequente em seus trabalhos. A falta de centralização dos dentes no filme pode inviabilizar a adequada visualização dos dentes radiografados e da área adjacente de interesse, como a região periapical, em razão destes estarem situados fora da área de registro do filme. Pontual et al. $(2005)^{12}$ observaram que erros de enquadramento foram os mais observados em sua amostra, quando os dados foram analisados de uma forma geral $(35,5 \%)$.

Radiografias com baixa ou alta 
densidade dificultam a visualização da imagem radiográfica pois perdem o contraste e a definição dos detalhes. Imagens pouco densas interferem na visualização de lesões radiopacas no periápice, restaurações infiltradas e a própria anatomia da câmara pulpar e canal radicular. Já imagens muito densas não favorecem o diagnóstico de lesões cariosas, imagens radiolúcidas no periápice, visualização da cortical alveolar e do espaço do ligamento periodontal. Baixa e alta densidade das radiografias foram, respectivamente, a terceira $(10,8 \%)$ e quinta $(9,9 \%)$ situações mais observadas. O $5^{\circ}(13 \%)$ e o $7^{\circ}(16,3 \%)$ períodos foram os que mais demonstraram radiografias com esses tipos de erros. A redução na densidade radiográfica foi o segundo erro mais observado por Carvalho et al. $(2009)^{6}$, terceiro por Matheus et al. $(2000)^{4}$ e o quarto no estudo de Mendonça et al. $(2014)^{13}$.

A presença de ranhuras foi o quarto erro mais observado na amostra $(9,9 \%)$, sendo o $10^{\circ}$ período aquele no qual se observou a maior ocorrência $(14,4 \%)$. Ranhuras nas radiografias reduzem substancialmente a sua qualidade para o diagnóstico e podem induzir a um diagnóstico errôneo de fratura radicular, por exemplo. No estudo de Queiroga et al. $(2010)^{5}$, a ocorrência de ranhuras foi o erro mais observado $(35,15 \%)$ enquanto Kreich et al. $(2002)^{2}$ constataram um percentual menor $(4,3 \%)$.

Radiografias amareladas ou manchadas por lavagem final insuficiente ou outros erros de processamento tornam-se completamente impróprias tanto para diagnóstico, quanto para documentação legal. Essas situações foram a sexta $(9,1 \%)$ e oitava $(5,7 \%)$ mais observadas, respectivamente. Radiografias amareladas foram mais observadas no $10^{\circ}$ período $(15,7 \%)$, enquanto outros erros de processamento foram mais detectados no $7^{\circ}$ período (10,5\%). Kreich et al. $(2002)^{2}$ observou uma grande frequência de radiografias amareladas $(18,3 \%)$. Pontual et al. $(2005)^{12}$ e Carvalho et al. (2009) ${ }^{6}$ apontaram o amarelamento como principal erro de processamento observado em seus estudos.

A sobreposição de faces proximais foi o sétimo erro mais observado na amostra $(7,5 \%)$. Esse erro decorre de angulação horizontal incorreta e dificulta, sobremaneira, a detecção radiográfica de lesões cariosas proximais. O período que mais apresentou esse erro foi o $6^{\circ}$ período $(17,4 \%)$. No estudo de Carvalho et al. (2009) ${ }^{6}$, o erro de angulação horizontal foi o mais frequente na amostra $(32,88 \%)$.

Frente às limitações que os erros impõem à interpretação radiográfica, as repetições de radiografias muitas vezes se tornam necessárias e demandam um maior tempo de trabalho ao profissional e maior exposição do paciente à radiação $\mathrm{X}$, além de custos desnecessários. Tavano e Álvares (2009) ${ }^{17}$ alertam quanto aos esforços para se evitar a repetição de exames por meio do uso da técnica correta e de processamento confiável e consistente. Para evitar erros, o exame radiográfico na Odontologia exige conhecimento e treinamento do cirurgiãodentista, o qual é iniciado durante a graduação. Mais estudos deveriam avaliar a frequência de erros de armazenamento dentre radiografias produzidas por estudantes de Odontologia, pois parece não haver dados quantitativos disponíveis na literatura, exceto os apresentados neste estudo.

A fim de se tentar reduzir as taxas de erros, um material informativo ilustrado foi confeccionado e afixado nas clínicas do curso de Odontologia da UFES. Esse material contém os resultados principais da presente pesquisa, com especial enfoque às medidas 
necessárias para o adequado armazenamento das radiografias periapicais nos prontuários do curso. O material visa informar não só os estudantes mas também os professores do curso, tendo em vista que, idealmente, as radiografias produzidas pelos estudantes necessitam passar pela avaliação dos professores de forma a assegurar a sua qualidade. Pretende-se, ainda, expor os resultados encontrados neste trabalho à comunidade acadêmica local sob a forma de palestras e reuniões de professores, de forma a promover o debate acerca do assunto e discutir estratégias para redução dos índices de erros.

\section{CONCLUSÃO}

Assim como em outras instituições de ensino superior, a taxa de radiografias com erros no curso de Odontologia da UFES é alta. $\mathrm{Na}$ avaliação realizada, armazenamento incorreto, falta de enquadramento e baixa densidade foram os erros mais encontrados. Deve-se atentar para o armazenamento de radiografias periapicais no âmbito universitário.

\section{Agradecimentos}

Agradecemos ao Conselho Nacional de Desenvolvimento Científico e Tecnológico (CNPq) pela bolsa de Iniciação Científica concedida para a realização do presente estudo.

\section{ABSTRACT \\ Technical errors in periapical radiographs taken by dental students of a Brazilian public university}

We aimed to identify and quantify the errors that dental students of UFES mostly commit when taking periapical radiographs. A total of 352 periapical radiographs was evaluated in this cross-sectional study. The radiographs were collected in the records of patients attended in the University in the last five years. Type of error and academic semester of the course were considered and data were submitted to the Chi-squared test at a significance level of $5 \%$. The results demonstrated a high frequency of errors $(84.37 \%)$ where the students in the $9^{\text {th }}$ academic semester showed the highest frequency $(92.30 \%)$ while those in the $8^{\text {th }}$ academic semester had the lowest $(70.00 \%)$ (p $<0.05)$. Improper storage $(15.60 \%)$ was the most frequent error in the sample and it has not been quantified for academic environment in the literature to the best of our science. The other two most frequent errors were: inadequate positioning of the film $(14.60 \%)$ and low radiographic density $(10.60 \%)$. We concluded that most of the periapical radiographs taken by the dental students of UFES have errors. Attention should be given to the storage of film-based periapical radiographs in universities.

Descriptors: Dental Students. Dental Radiographs. Quality Control.

\section{REFERÊNCIAS}

1. Whaites E. Princípios de Radiologia Odontológica. $4^{\mathrm{a}}$ ed. Rio de Janeiro: Elsevier, 2009.

2. Kreich EM, Queiroz MGS, Sloniak MC. Controle de qualidade em radiografias periapicais obtidas no curso de Odontologia da UEPG. Publ UEPG Ci Biol Saúde. 2002; 8(1):33-45.

3. Fernandes AMM, Aguiar APS, Cruz LP, Aivazoglou MU, Gomes APM, Silva EG, et al. Avaliação dos erros radiográficos cometidos por alunos de graduação durante o tratamento endodôntico. Rev Odontol Univ Cid São Paulo. 2010;22(3):216-22.

4. Matheus RA, Montebel Filho A, Tanaka EE, Barros RMP. Avaliação no desempenho de acadêmicos durante tomadas radiográficas periapicais, pela técnica da bissetriz, realizadas na Faculdade de Odontologia da 
Universidade Norte do Paraná (Unopar). RFO UPF. 2000;5(2):25-9.

5. Queiroga MAS, Moreno NPP, Figueiredo CBO, Abreu MHNG, Brasileiro CB. Avaliação dos erros radiográficos cometidos por graduandos de odontologia em técnicas radiográficas intrabucais. Arq Odontol. 2010;46(3):61-5.

6. Carvalho PL, Neves ACC, Zollner NA, Rosa LCL, Almeida ETDC. Erros técnicos nas radiografias intrabucais realizadas por alunos de graduação. RGO. 2009;57(2):151-5.

7. Brasil. Resolução CNE/CES 3/2002. Diário Oficial da União, Brasília, 4 de março de 2002. Seção 1, p.10. Disponível em: http://portal.mec.gov.br/cne/arq uivo/pdf/CES32002.pdf

8. Brasil. Ministério da Saúde. Secretaria de Vigilância Sanitária. Diretrizes de Proteção Radiológica em Radiodiagnóstico Médico e Odontológico. Portaria $\mathrm{N}^{\mathrm{o}} 453$, de $1^{\circ}$ de junho de 1998. Disponível em: http://www.cro-rj.org. br/radiologia/PF453integra.pdf

9. White SC, Pharoah MJ. Radiologia oral: fundamentos e interpretação. $7^{a}$ ed. Rio de Janeiro: Elsevier; 2015.

10. Freitas A, Rosa JE, Souza IF. Radiologia odontológica. $7^{\mathrm{a}}$ ed. São Paulo: Artes Médicas; 2008.

11. Ribas ME, Souza BC, Fontanella VRC. Avaliação da qualidade de radiografias intrabucais realizadas por acadêmicos da FO-UFRGS. Rev ABENO. 2008;8(1):4950.
12. Pontual MLA, Veloso HHP, Pontual AA, Silveira MMF. Errores en radiografias intrabucales realizadas en la Facultad de Odontología de Pernambuco-Brasil. Acta Odontol Venez. 2005; 43(1):19-24.

13. Mendonça DM, Amorim MEB, Medina PO, Filho AOA, Brito TCCA, Conde NCO. Avaliação de erros em exames radiográficos intrabucais realizada por acadêmicos de Odontologia/UFAM. Rev Odontol Univ Cid São Paulo. 2014;26(2):119-26.

14. Gonçalves A, Bóscolo FN, Gonçalves M. Análise de erros radiográficos cometidos por alunos de faculdade de odontologia durante o tratamento endodôntico. RFO UPF. 2003; 8(1):61-5.

15. Helminen SE, Vehkalahti M, Wolf J, Murtomaa H. Quality evaluation of young adults' radiographs in Finnish public oral health service. J Dent. 2000;28(8):549-55.

16. Ferreira LPF, Santos CE. A qualidade da radiografia e erros mais comuns na auditoria radiográfica. Braz $\mathrm{J}$ Forensic Sci Med Law Bioethics. 2015;4(3):28893.

17. Tavano O, Alvares LC. Curso de Radiologia em Odontologia. $5^{\text {a }}$ ed. São Paulo: Santos; 2009.

Correspondência para:

Sergio Lins de Azevedo Vaz

e-mail: sergiolinsv@ gmail.com

Departamento de Clínica Odontológica CCS/UFES

Av. Marechal Campos, 1468

29043-900 - Maruípe, Vitória/ES, 